\title{
Vaccine needed for Aboriginal children
}

$\mathrm{A}$

10-month-old boy, admitted to hospital in Saskatchewan in November 2013 with a bacterial infection in his blood, did well on antibiotics and returned home, but a few months later he was back in hospital with meningitis and this time, "despite vigorous antibiotic and supportive treatment," he died. The culprit in both his infections was the bacterium Haemophilus influenza serotype a (Hia). For Raymond Tsang, a laboratory chief with the Public Health Agency of Canada's National Microbiology Laboratory, the baby's death underscores the seriousness of the infection, especially for young Aboriginal children in Canada's North, and the need for a vaccine.

Type b Haemophilus influenza (Hib), once a leading cause of childhood meningitis, was nearly wiped out when the Hib vaccine was added to childhood immunizations in the early 1990s. Now Hia is causing very serious infections in children in the North, including meningitis and bacteremia.

Animal tests of a prototype vaccine are promising, but funding is scarce and commercial co-partners scarcer still. Though scientists who track bacterial disease are on the alert for Hia, there is little awareness of it among health care professionals.

Physicians often confuse the infection with influenza, says Dr. Marina Ulanova, an immunologist at Northern Ontario School of Medicine (NOSM) in Thunder Bay who has been studying Hia for eight years. "People who work in health care very often don't even know what it is."

Part of the awareness problem stems from the fact that Canada-wide data on Hia is impossible to obtain. Although Hia became a reportable disease in Canada in 2007, not everyone follows the guidelines. Ontario and some other affected provinces do not systematically perform surveillance of Hia. The infection may also be off the radar of granting agencies: Canadian Institutes of Health Research (CIHR)

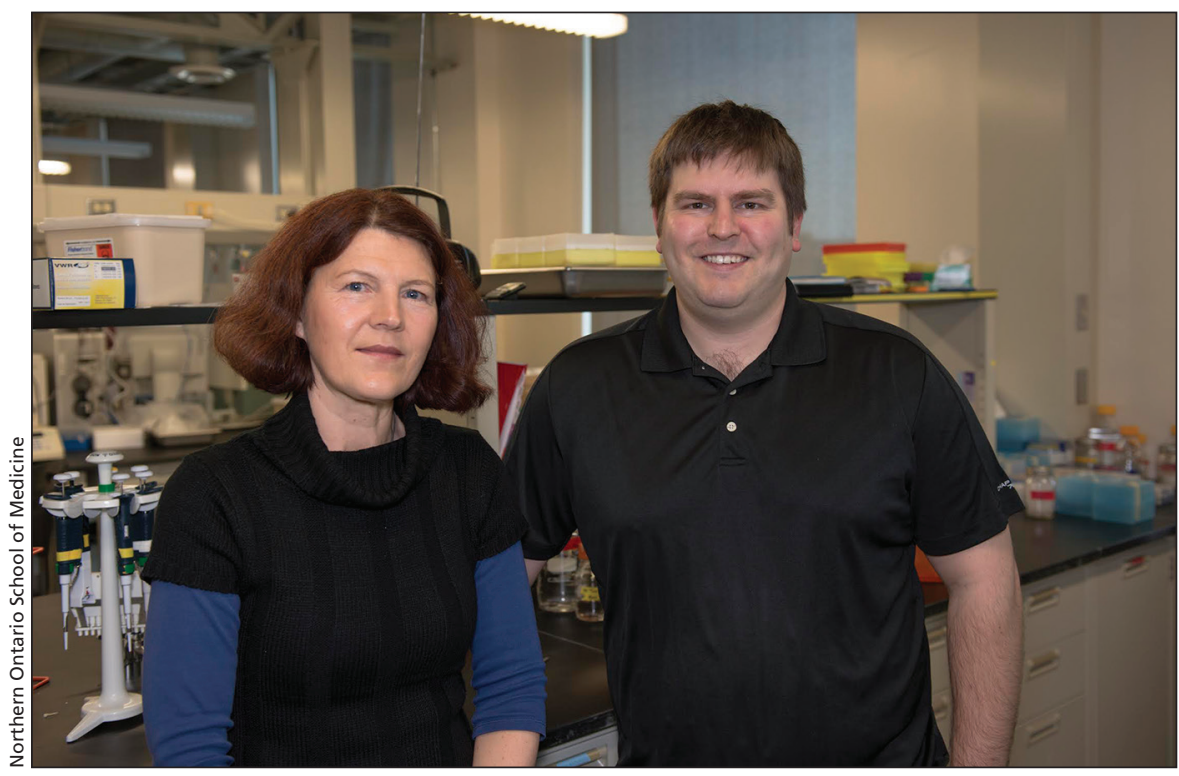

Dr. Marina Ulanova and Eli Nix's efforts into developing and Hia vaccine are frustrated by a lack of funding.

has declined to fund Ulanova's work on Hia four times.

But there is evidence that Hia is taking a toll. Dr. Shalini Desai of Public Health Ontario reported at a recent conference that since Canada's circumpolar region, an area that includes parts of five provinces, started surveillance of Hia in 2000 , the number of cases has steadily increased. As of 2013, the region had documented a total of 81 children under age five with Hia in the 13-year period. Of those, 40 were under age one and nearly all were Aboriginal. Over $80 \%$ of the children required admission to hospital; many had to be transferred out of province for care. Six died.

Other reports of infected children come from British Columbia, Manitoba, and Northwest Ontario. Ulanova, Tsang and others have published descriptions of patients treated for Hia at Thunder Bay Regional Health Sciences Centre, Meno Ya Win Health Centre in Sioux Lookout and a health centre in Kenora, including two children who were hospitalized for weeks with both pneumonia and meningitis, and several adults whose infections came on top of chronic illnesses such as diabetes.
This evidence is sufficient to prompt a vaccine effort by NOSM, the Public Health Agency of Canada (PHAC) and the National Research Council of Canada (NRC). "I'm very satisfied that we've got a candidate vaccine in hand," says Dr. Matthew Gilmour, PHAC's new scientific director general.

The United States Centers for Disease Control and Prevention (CDC) is also involved in the vaccine project because of Hia in Alaska, where Dr. Michael Bruce of the CDC's Arctic Investigations Program says it is a "major cause of meningitis in Alaskan Native children now." More than 40 Alaskans, mostly Native children under age five, have had the infection since 2002; three infants have died.

The idea is to develop a vaccine similar to the Hib vaccine, which Andrew Cox, a research officer in the NRC's Vaccine Program, terms "the gold standard." The Hib vaccine is based on the type b bacteria's outer carbohydrate coat or capsule, so NRC scientists followed "relatively well known methods" to isolate the carbohydrate capsule for type a, Cox says. They then purified it, chemically modified it to attach it to a 
bacterial protein and tested it at the NRC's animal facility, where they successfully immunized mice and rabbits, though they haven't yet published the work.

The next step, finding a pharmaceutical company to manufacture the vaccine for clinical trials, is a huge hurdle. Firms are not showing interest in a vaccine that will be targeted to vulnerable groups and is unlikely to be lucrative.

"One of the critical pieces is establishing the public health need for this vaccine," says Gilmour. To that end, the agency is working with provincial laboratories and international partners, such as the Pan American Health Organization, to increase surveillance for Hia in Canada and Latin America.

\section{Why the risk?}

The question of why Aboriginals are at greatest risk remains an open one. Eli Nix, a CIHR postdoctoral fellow in Ulanova's lab at NOSM, investigated levels of Hia immunity in Northwest Ontario and the results shifted his focus away "from the idea that it's some sort of deficiency or weakness in First
Nations people that's making them vulnerable."

Among adults in Thunder Bay, he found that Aboriginals have more potent antibodies against Hia compared with non-Aboriginals, which suggests that "it's maybe geography," he says. "Maybe the bacteria happen to be in a certain area." Aboriginal adults could have more functional antibodies against Hia because they encounter the bacteria more often, with young children more likely to get sick from it. Nix published his findings in February in Emerging Infectious Diseases.

$\mathrm{He}$ and Ulanova are also conducting studies considered crucial to proceeding with a vaccine, comparing Hia antibody levels in a First Nations community in Northern Ontario to the levels in a First Nations community further south in Ontario's Bruce County, where the incidence is much lower. But progress is slow; it took two years to find communities willing to participate. Nix, who is Algonkian of Pikwàkanagàn, says he understands the reluctance due to "residential schools and the kind of injustices and things that have gone on in the past," but he admits he was frustrated.

Nix is starting medical school at NOSM in the fall and Ulanova is unsure if she can continue the work. "I have zero funding for this."

The vaccine project is also at a standstill, lacking an industry partner. NRC staff have spoken with the Bill \& Melinda Gates Foundation and the global vaccine fund GAVI, though NRC's Andrew Cox thinks that would be an unusual path to take.

"My query would be that at the end of the day, it's still a concern for Canada," he says, "and Canada is relatively a wealthy country so we should be able to look after our own, so I don't know whether that approach would fly."

But the vaccine project is not yet at the point where the government will step in, Gilmour says. "We're certainly not at a stage where we can walk on Ottawa, so to speak, and just ask that this gets made. We're going to have to build the evidence to say that's true." Miriam Shuchman MD, Toronto, Ont.

CMAJ 2015. DOI:10.1503/cmaj.109-5047 